\title{
EDITORIAL
}

\section{Analytical Quality by Design}

\section{Márcia Cristina Breitkreitz (iD $ه$}

Guest Editor of this special edition on Chemometrics devoted to Prof. Ronei J. Poppi

Professor at the Institute of Chemistry, University of Campinas

Campinas, SP, Brazil

Throughout the $20^{\text {th }}$ century, the notion of 'quality' underwent major changes. The concept of 'Quality by Testing', i.e. evaluating the quality of a product by testing it for some pre-defined parameters after completing the manufacturing process, started to be replaced by the enhanced approach of Quality by Design (QbD). In this concept, idealized by Joseph Juran [1], quality is initially conceived for the product, and then the product is manufactured and evaluated to reach that quality. Driven by the need to reduce costs and to encourage companies to improve their understanding of their products and manufacturing processes, pharmaceutical regulatory agencies, through the $\mathrm{ICH}$ (International Council on Harmonization), published the ICH Q8 guideline, highlighting the QbD strategy for pharmaceutical development [2]. This represented a significant step in replacing the existing quality paradigms in the conventional manufacturing of pharmaceutical products and paved the way to support development and production activities within a scientific, flexible environment, with a high level of quality, without the need for extensive regulatory surveillance. The higher level of understanding gained during development allows out-of-specification batches to be foreseen and ensures that the desired quality will be obtained at the end of the manufacturing process. The two pillars of the QbD concept are quality risk management and multivariate study of the outcomes (quality parameters) as functions of the inputs (materials, formulation and process inputs). At this point, Chemometrics emerged officially as a strategy to support the pharma regulatory requirementsthe beginning of a joyful and long-life union.

Soon analytical researchers realized that the QbD concepts could be readily applied to the analytical method development, since it is also a 'process' with quality requirements to be fulfilled. This generated what is nowadays called 'Analytical Quality by Design' (AQbD) [3-5]. The development of increasingly efficient analytical methods presents itself as a fundamental aspect in the pharmaceutical industry, since the analysis of drugs in raw materials, intermediates and finished products is necessary in practically the entire process of the development and production of medicines. High Performance Liquid Chromatography (HPLC) is the most commonly used technique in the pharmaceutical industry [6], along with the modern version of Ultra High Performance Liquid Chromatography (UHPLC). Therefore, the majority of efforts in $\mathrm{AQbD}$ are directed towards these techniques.

In the same way as with $\mathrm{QbD}, \mathrm{AQbD}$ is driven by quality risk management and understanding of the quality parameters of the method as functions of the inputs (experimental) variables. To implement the principles of $A Q b D$, one must first define all of the requirements that the method must meet in terms of quality; that is, define the Analytical Target Profile (ATP), which includes both procedure attributes and validation parameters, depending on the purpose of the method and the guidelines being followed. From the ATP, the attributes that are critical for the proper performance of the method should be identified; these are called Critical Quality Attributes - CQA. Some examples of CQA include the adequate retention in the column (measured by the retention factor, k); appropriate resolution among all target peaks in the chromatogram (measured by the resolution $\mathrm{R}_{\mathrm{s}}$ or separation factor, $\alpha$ ); adequate symmetry for integration

Cite: Breitkreitz, M. C. Analytical Quality by Design. Braz. J. Anal. Chem., 2021, 8 (32), pp 1-5. doi: http://dx.doi.org/10.30744/ brjac.2179-3425.editorial.mcbreitkreitz.N32 
(measured by tailing factor or an equivalent parameter); plate number; number of peaks observed in the chromatogram, etc. In addition, a target value or acceptance limit must be established for each of them.

The next step involves identifying the critical experimental variables that influence these quality attributes, using prior knowledge and risk assessment tools (e.g. Ishikawa diagram), and then assigning a risk to each of them - which can be done either by a formal risk analysis tool such as FMEA (Failure Mode Effect Analysis) or through risk analysis tables. An important aspect is the justification of the risk assigned to each variable according to analytical knowledge of the samples being analyzed and the analytical technique used. The high-risk variables are called Critical Procedure Parameters (CPP) or Critical Method Parameters (CMP) and some examples are: column type (chemical composition of the stationary phase and column length), composition of the mobile phase (type of organic modifier, $\mathrm{pH}$ of the aqueous phase, ion-pairing reagent type), gradient slope or gradient time, temperature of the column, flow rate, etc.

The $\mathrm{QbD}$ strategy recommends the study of the CQA as a function of the CPP in a multivariate way and, for this reason, the tools of Design of Experiments - (DOE) are fundamental in this strategy. This procedure has several advantages over the univariate OFAT (One-factor-at-a-time) method, which is normally timeconsuming and often does not bring satisfactory solutions, due to interactions of the variables involved (i.e., the response obtained by changing variable A depends on the level of variable B), which can only be identified using multivariate methods. In addition, in the OFAT method, the results are only obtained at the points where the experiments are carried out, not allowing characterization of the entire experimental domain. On the other hand, multivariate methods may allow an understanding of how the CQA changes depending on the variation of the levels of CPP through the estimation of mathematical models which allow the visualization of results by plotting response surfaces, and the prediction of responses within the experimental domain without the need for extra laboratory work.

Since chromatographic method development involves many variables, different DOE methods can be used according to the nature of these variables (statistically independent/non-independent variables), the goal of the experiment and the number of variables involved. As a general practical guide, the primary variables that affect selectivity in liquid chromatography - type of stationary phase, nature of organic modifier (typically methanol (acidic property), acetonitrile (dipolar) or tetrahydrofuran (basic) in reversed phase liquid chromatography) and $\mathrm{pH}$ range (acidic, neutral, basic ranges) - should be screened in the first step of method development. Since initial guidance is the objective of this step, economical designs, such as fractional factorial or optimal designs are recommended. Optimal designs are versatile computergenerated designs that set the experiments according to a given pre-defined mathematical criterion, for example, D-, G-, A-, I-optimality criteria. They are especially welcome in situations when constraints need to be imposed on the design region, when a nonstandard model is necessary to adequately explain the response or when the number of experiments should be restrained to estimate a pre-defined model [7].

A second step encompasses the use of design methods to estimate the coefficients of a prediction model, with pre-defined CQA as responses, and temperature, flow rate, gradient time (or slope) and $\mathrm{pH}$ (in a narrower range) typically being the input variables. For this purpose, Full Factorial, Central Composite, Box-Behnken, Doehlert and optimal designs can be used. It should be highlighted that Full Factorial designs only allow the estimation of linear models, whereas the other methods mentioned allow higher order coefficients to be estimated. Mixture designs can be used for optimization of the mobile phase composition, considering solvents with different characteristics as the mixture ingredients - the selection can be made using the well-known Snyder's selectivity triangle for liquid chromatography. A detailed description of DOE methods applied to chromatography can be found in many references [8-11].

In order to ensure that quality parameters are achieved for all compounds in the same chromatographic run, a procedure for the simultaneous optimization of several responses can be employed. Even though many 'response functions' attempt to describe the overall separation by a single value, experience shows that the best way is to model individual responses and then combine them using a multiple response optimization method, such as the contour map overlay and the desirability functions of Derringer and Suich [12-13]. These methods allow a very important precept of $Q b D$ to be achieved, which is the 'Design Space' 
(DS) or 'Method Operable Design Region' (MODR), defined as the "multidimensional combination of variables that influence a method and that guarantee the quality of the data produced by it" [14]. According to this definition, the MODR is considered a robust work region, since the experimental variations within this region do not cause changes in the quality attributes of the method. It is worth mentioning that, at this stage, a careful statistical analysis of the mathematical models from the selected DOE that generated the MODR must be done, using, for example, analysis of variance (ANOVA), confidence intervals and analysis of residuals.

The major benefit of building an MODR is to incorporate the robustness into the development, instead of evaluating it in the validation step, as is currently done. This avoids undesirable surprises during validation and may allow changes to be made over the method's lifecycle in a safe way, without the need for regulatory approval $[15,16]$. An important step of increasing interest is the evaluation of MODR uncertainty limits. Monte-Carlo simulations, Bayesian modelling and bootstrapping are useful tools to accomplish this task and ensure the robustness within the MODR $[17,18]$. Estimates of confidence intervals for the predicted responses also represent a way to take into account the uncertainty of MODR.

A concrete proof that this approach is the future of analytical development by liquid chromatography is the launching of commercial software for method optimization and data treatment, already connected to or integrated with analytical instruments. The most representative example is Fusion QbD LC method development (S-Matrix, California, EUA) which provides a full range of DOE tools to support liquid and supercritical fluid chromatography method development and which is integrated in the software of major instrument manufacturers, such as Empower (Waters Technologies), Chromeleon (Thermo Scientific) and OpenLab Chem Station (Agilent). Fusion QbD mainly works with optimal designs (other designs are also available) to select the minimum number of runs to obtain information in a two-step approach - Screening and Optimization. The sequence of runs is organized by the software and information is exported to the instrument. After completion, chromatographic peaks should be integrated by the user and the results exported back to Fusion. Then, the software allows a complete statistical data analysis, including model selection and evaluation by ANOVA and residual graphs; response surfaces are built and desirability functions combined with Monte Carlo simulations allow the MODR to be determined with uncertainty boundaries. Also, predicted chromatograms can be plotted in real-time according to the variations made within the studied experimental region. This certainly represents the state-of-the art in the LC method development.

Another outcome that highlights the importance of $A Q b D$ is the fact that this strategy has been recognized by official entities all over the world. In June 2019, the British Medicines and Healthcare products Regulatory Agency (MHRA) published the results of a public consultation on the use of AQbD principles to pharmacopeial standards for medicines. This was accompanied by the publication of a technical review of an MHRA project to explore the application of AQbD to a pharmacopeial assay procedure [19]. Responses to that public consultation declared that the MHRA will support and complement the evolution of developing regulatory science, with the application of AQbD concepts [20]. The United States Pharmacopeia (USP) has published a proposed new General Chapter $<1220>$ The Analytical Procedure Lifecycle aiming at describing the current thinking of the USP Validation and Verification Expert Panel which advises the General Chapters - Chemical Analysis Expert Committee with regard to future trends in analytical procedure development, qualification, and continued monitoring. This general chapter article had the purpose of describing an alternative approach to the classical process of analytical validation and subsequent verification and transfer, describing these activities as a continuum and closely interrelated events rather than as discrete actions [21]. This is only possible by using AQbD concepts, in particular modelling of the CQA as functions of the CPP and the establishment of the MODR. A similar document is expected to be published this year by the ICH for public consultation, the ICH Q14 Analytical Procedure Development guideline, which is based on the principles of the ICH Q8, i.e. sound scientific and risk-based approval, as well as post-approval change management, now for analytical procedures [22]. 


\section{CONCLUSIONS AND FUTURE PERSPECTIVES}

The strategy of varying the experimental conditions in a univariate way and selecting the "best chromatogram" is becoming unacceptable within the context of modern analytical chemistry and quality environments. The use of DOE and the application of multivariate methods to method development are key elements in the context of the Analytical Quality by Design initiative - these will be strictly necessary tools for analytical development in the future, and can no longer be avoided, especially after being adopted by regulators. The development of methods using the univariate strategy, in addition to being very timeconsuming, does not always bring satisfactory solutions, because interactions can occur between the many variables involved, which can only be identified using multivariate methods. In addition, results are only obtained at the points where the experiments were carried out, not allowing an overview of the complete experimental domain. In the current method development strategy, robustness is only tested in the validation step; nevertheless, once this stage is reached, one may realize that the method is not robust even to small fluctuations in the experimental conditions meaning that it is necessary to go back to the development stage. With the proposed strategy, the robustness is naturally incorporated into the method development stage.

\section{Acknowledgments}

I would like to thank Prof. Dr. Roy Edward Bruns and Prof. Dr. Isabel Cristina Sales Fontes Jardim, my Masters advisors (2004-2007) when I started my studies on DOE applied to chromatography, already knowing it would be something very important in the future. Also thanks to Amanda Guiraldelli Mahr (USP Pharmacopoeia) and Richard Verseput (S-Matrix) for the current partnership in AQbD projects - and very nice discussions.

Finally, I would like to thank and dedicate this Editorial to Prof. Dr. Ronei Jesus Poppi, who was my PhD advisor and became a friend, counselor and example much beyond Chemometrics.

\section{REFERENCES}

1. Juran, J. M. Juran on Quality by Design: The New Steps for Planning Quality Into Goods and Services. Free Press, New York, 1992, Chapter 2, p 27.

2. International Council on Harmonization. ICH Q8 (R2) Pharmaceutical development, 2009.

3. Vogt, F. G; Kord, A. S. J. Pharm. Sci., 2011, 100 (3), pp 797-812 (https://doi.org/10.1002/jps.22325).

4. Orlandini, S.; Pinzauti, S.; Furlanetto, S. Anal. Bioanal. Chem., 2013, 405, pp $443-450$ (https://doi. org/10.1007/s00216-012-6302-2).

5. Peraman, R.; Bhadraya, K.; Reddy, Y. P. Int. J. Anal. Chem., 2015, 2015, pp 1-9 (http://dx.doi. org/10.1155/2015/868727).

6. Siddiqui, M. R.; AlOthman, Z. A.; Rahman, N. Arabian J. Chem., 2017, 10, pp 1409-1421 (http://dx.doi. org/10.1016/j.arabjc.2013.04.016).

7. Johnson, R. T.; Montgomery, D. C.; Jones, B. A. Qual. Eng., 2011, 23, pp 287-301 (https://doi.org/10. 1080/08982112.2011.576203).

8. Ferreira, S. L. C.; Bruns, R. E.; da Silva, E. G. P.; dos Santos, W. N. L.; Quintella, C. M.; David, J. M.; de Andrade, J. B.; Breitkreitz, M. C.; Jardim, I. C. S. F.; Neto, B. B. J. Chromatogr. A, 2007, 1158, pp 2-14 (https://doi.org/10.1016/j.chroma.2007.03.051).

9. Hibbert, D. B. J. Chromatogr. B., 2012, 910, pp 2-13 (https://doi.org/10.1016/j.jchromb.2012.01.020).

10. Dejaegher, B.; Heyden, Y. V. J. Pharm. Biomed. Anal., 2011, 56, pp 141- 158 (https://doi.org/10.1016/j. jpba.2011.04.023).

11. Bezerra, M. A.; Santelli, R. E.; Oliveira, E. P.; Villar, L. S. Escaleira, L. A. Talanta, 2008, 76, pp 965-977 (https://doi.org/10.1016/j.talanta.2008.05.019).

12. Derringer, G.; Suich R. J. Qual. Tech., 1980, 12, pp 214-219 (https://doi.org/10.1080/00224065.1980. 11980968).

13. Candioti, L. V.; De Zan, M. M.; Cámara, M. S.; Goicoechea, H. C. Talanta, 2014, 124, pp 123-138 (https://doi.org/10.1016/j.talanta.2014.01.034). 
14. Rozet, E.; Lebrun, P.; Debrus, B.; Boulonger, B.; Humbert, P. TrAC, Trends in Anal. Chem., 2013, 42, pp 157 - 167 (https://doi.org/10.1016/j.trac.2012.09.007).

15. Hubert, C.; Houari, S.; Rozet, E.; Lebrun, P.; Hubert, Ph. J. Chromatogr. A, 2015, 1395, pp 88-98 (http://dx.doi.org/10.1016/j.chroma.2015.03.059).

16. Chatterjee, S. IFPAC Annual Meeting, Baltimore, January 25, 2013. Available at: http://qbdworks.com/ wp-content/uploads/2014/06/AnalyticalQbDIFPAC.pdf [Acessed March 2021].

17. Deidda, R.; Orlandini, S.; Hubert, P.; Hubert, C. J. Pharm. Biomed. Anal., 2018, 161, pp 110-121 (https://doi.org/10.1016/j.jpba.2018.07.050).

18. Dispas, A.; Avohou, H. T.; Lebrun, P., Hubert, P.; Hubert, C. TrAC, Trends Anal. Chem., 2018, 101, pp 24-33 (https://doi.org/10.1016/j.trac.2017.10.028).

19. Medicines \& Healthcare products Regulatory Agency (UK). Consultation: Application of Analytical Quality by Design concepts to pharmacopoeial standards for medicines, 2019. Available at: https:// assets.publishing.service.gov.uk/government/uploads/system/uploads/attachment_data/file/806198/ AQbD_Consultation_document.pdf [Acessed March 2021].

20. Medicines \& Healthcare products Regulatory Agency (UK). Consultation response: MHRA response and Strategy for the Application of Analytical Quality by Design concepts to pharmacopoeial standards formedicines, 2020. Available at: https://assets. publishing.service.gov.uk/government/uploads/system/ uploads/attachment_data/file/908703/AQbD_Consultation_Public_response_Final.pdf [Acessed March 2021].

21. Stimuli to the revision process USP Validation and Verification Expert Panel. Available at: http:// www.ichq3d.fr/files/supplier/fichiers/2/43(1)\%20Stimuli\%20to\%20the\%20Revision\%20Process:\%20 Proposed\%20New\%20USP\%20General\%20Chapter:\%20The\%20Analytical\%20Procedure\%20Li. pdf [Acessed March 2021].

22. International Council for Harmonisation of Technical Requirements for Pharmaceuticals for Human Use (ICH). ICH Q14. Analytical Procedure Development and Revision of Q2(R1) Analytical Validation, 2018. Available at: https://database.ich.org/sites/default/files/Q2R2-Q14_EWG_Concept_Paper.pdf [Acessed March 2021].

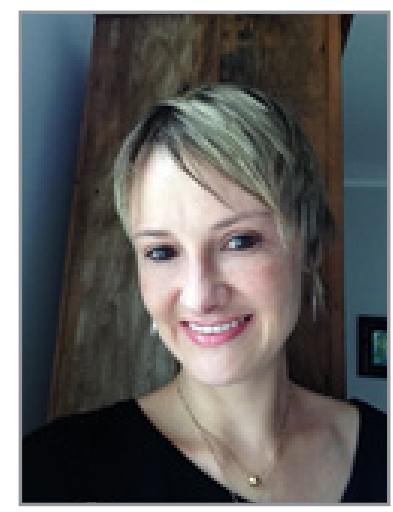

Márcia Cristina Breitkreitz has been working since 2014 as Professor in the Chemistry Institute of the University of Campinas (UNICAMP), coordinating the Laboratory of Pharmaceutical Research and Chemometrics (LabFarQui). She was a senior researcher in Chemometrics from 2006-2007 at the International Institute of Pharmaceutical Research (IIPF, Brazil) and a coordinator of research and development from 2007 to 2009 in the same Institute. She received her PhD in Chemistry from UNICAMP in 2013, under the supervision of Prof. Ronei Jesus Poppi. Her major research interests are related to Chemometrics applied to the development of pharmaceutical products and processes according to the strategy of Quality by Design (QbD); development of analytical methods based on liquid/ supercritical fluid chromatography and vibrational spectroscopy (Raman and NIR) for pharmaceuticals. In addition to her research work and teaching (graduate and undergraduate levels), Prof. Marcia is dedicated to courses and consultancy on relevant topics to the pharmaceutical industry, especially related to Chemometrics within the context of Quality by Design. 